questionnaire in the week preceding the event. 11809 runners completed the survey.

Interventions (or Assessment of Risk Factors) Demographics including age, gender, experience and training history.

Results The average age of respondents was 40.9y (range 18$83 y)$ and $54.1 \%$ were male. $22.3 \%$ of respondents developed a new illness in the 4 weeks prior to the event. Upper respiratory tract infection (URTI) was most common (64.3\%), followed by GI problems (15.4\%) and headache/migraine (14.6\%). 28.5\% of respondents who had been training for $<2$ months developed an acute illness, compared with $19.8 \%$ of those trained for $>6$ months $(p=0.0002)$. Lower average weekly training distance $(22.9 \%$ of those training from $<20$ to 40 miles/week vs. $18.7 \%$ training from 40 to $>50$ miles/ week; $\mathrm{p}<0.05)$ and shorter longest training run $(24.4 \%$ whose longest run was $<20$ miles vs. $19.4 \%$ whose longest training run was $>20$ miles; $\mathrm{p}<0.05$ ) were associated with higher incidence of acute illness. $25.0 \%$ of novice runners (running $<1 \mathrm{y}$ ) developed an acute illness compared to $20.3 \%$ of those who had been running $>10 y(p<0.05)$.

Conclusions Novice runners who train for $<2$ months with low average weekly training mileage were more likely to develop an acute illness during marathon training than more experienced runners. Further research is needed to establish the direction and relationship between these factors before guidance can be issued.

\section{WHAT ARE THE MAIN RISK FACTORS FOR LOWER- EXTREMITY RUNNING-RELATED INJURIES? A RETROSPECTIVE SURVEY-BASED ON 3669 RESPONDENTS}

\footnotetext{
1,2Damien Sanfilippo, ${ }^{1}$ Charlotte Beaudart, ${ }^{1}$ Olivier Bruyère, ${ }^{1,2}$ Jean-François Kaux, ${ }^{2}$ Géraldine Martens. 'University and University Hospital of Liège, Liège, Belgium; ${ }^{2}$ ReFORM, Research Centre for the Prevention of Injury and IIIness and the Protection of Athletes, Liège, Belgium
}

\subsection{6/bjsports-2021-IOC.303}

Background Many studies attempt to identify the risk factors for running-related injuries (RRI), but these are not yet well established.

Objectives To investigate the risk factors of RRI.

Design Retrospective online survey-based study among population of runners injured and non-injured.

Setting Leisure road and trail runners

Patients Participants have to be at least 18 years old and have to practice running at least for 12 months. 3669 runners reported information which were included for statistical analysis.

Assessment of Risk Factors The online survey included 41 questions with five main categories: personal characteristics daily lifestyle- training and running characteristics - practice of others sports activities and prevention habits.

Main Outcome Measurements Occurrence of running-related injury over the last 12 months.

Results Amongst the 3669 runners, 1852 (50.5\%) reported at least one injury over the last 12 months. Overuse injury were largely represented (60.6\%). The variables associated with RRI which remained significant in the fully-adjusted model were: previous injury $(\mathrm{OR}=1.63$, IC 95\% $=1.42-1.47)$, competition running $(\mathrm{OR}=1.62$, IC $95 \%=1.26-2.09)$, more than 2 hours running per week $(\mathrm{OR}=1.30$, IC 95\%= $1.03-1.65)$, mileage $(>20 \mathrm{~km} /$ week $)(\mathrm{OR}=1.25$, IC $95 \%=1.01-1.55)$ and speed training $(\mathrm{OR}=1.23$, IC 95\%=1.06-1.48). Univariate analysis revealed other variables associated with more RRI: Trail runners (versus road runners, $\mathrm{p}<0.001$ ), men (versus women, $\mathrm{p}<0.001)$, higher age $(\mathrm{p}<0.001)$, $>2$ running session/week $(\mathrm{p}<0.001)$.

Conclusions Previous injury remains the most relevant RRI risk factor according to the current study and previous data. Many training characteristics seem to be involved but still have to be confirmed in view of conflicting data in literature. Trail runners are more at risk of RRI. Further research would help to understand better RRI and to prevent them.

\section{EPIDEMIOLOGY OF INJURY AND ILLNESS AMONG TRAIL RUNNERS: A SYSTEMATIC REVIEW}

${ }^{1}$ Carel Viljoen, ${ }^{2}$ Christa Janse van Rensburg, ${ }^{3}$ Evert Verhagen, 4,5,6,7 Willem van Mechelen, ${ }^{8}$ Rita Tomas, ${ }^{10}$ Marlene Schoeman, ${ }^{9}$ Susan Scheepers, ${ }^{1}$ Elzette Korkie. ${ }^{1}$ Department of Physiotherapy, Faculty of Health Sciences, University of Pretoria and Sport, Exercise Medicine and Lifestyle Institute (SEMLI), Pretoria, South Africa; ${ }^{2}$ Sport, Exercise Medicine and Lifestyle Institute (SEMLI) and Section Sports Medicine Faculty of Health Sciences, University of Pretoria, Pretoria, South Africa; ${ }^{3}$ Amsterdam Collaboration for Health and Safety in Sports, Department of Public and Occupational Health, Amsterdam Movement Sciences, VU University Medical Center, Amsterdam, Netherlands; ${ }^{4}$ Amsterdam Collaboration for Health and Safety in Sports, Department of Public and Occupational Health, Amsterdam Movement Sciences, VU University Medical Center, Amsterdam, The Netherlands, Amsterdam, Netherlands; ${ }^{5}$ School of Human Movement and Nutrition Sciences, Faculty of Health and Behavioural Sciences, University of Queensland, Brisbane, Australia; ${ }^{6}$ Division of Exercise Science and Sports Medicine (ESSM), Department of Human Biology, Faculty of Health Sciences, University of Cape Town, Cape Town, South Africa; ' School of Public Health, Physiotherapy and Population Sciences, University College Dublin, Dublin, Ireland; ${ }^{8}$ Portugal Football School, Portuguese Football Federation, Lisbon, Portugal; ${ }^{9}$ Department of Library Services, Faculty of Health Sciences, University of Pretoria, Pretoria, South Africa; ${ }^{10}$ None, Pretoria, South Africa

\subsection{6/bjsports-2021-IOC.304}

Background Trail running (TR) is characterised by uneven varying running surfaces, with large elevation gains/losses. Therefore, the injury and illness profiles of TR may differ compared to road running. Limited information is available on injury and illness among trail runners (TRs) to help develop interventions towards injury prevention.

Objective Systematically review data on TR injury and illness.

Design Systematic review.

Setting MEDLINE Ovid, PubMed, Scopus, SportsDiscus, CINAHL, Health Source: Nursing/Academic, Health Source: Consumer Ed. and Cochrane were searched from inception to February 2019. Methodological quality was assessed using an adapted Downs and Black assessment tool.

Patients (or Participants) N/A (systematic review abstract).

Interventions (or Assessment of Risk Factors) Studies were included if they investigated injury and/or illness among TRs participating in training/racing and full-text available in English/French. Studies were excluded on biomarkers of injury/illness in the absence of participants reporting injury/illness, or if no clear evidence was found of investigating TR.

Main Outcome Measurements TR injury (incidence, prevalence, anatomical site, tissue type, specific diagnosis, severity) and illness (incidence, prevalence, symptoms, specific diagnosis, body system, severity).

Results Fourteen studies with 3094 participants were included. Six studies investigated injuries and illnesses, 3 studies investigated only injuries and 5 studies only illnesses. Twelve studies investigated race-related injury and/or illness and 2 studies 
included training-related injuries. Different study designs, injury and illness definitions, race distances, and surfaces, made pooling of results difficult. The foot, knee, ankle and thigh are the most common anatomical sites of TR injury, with lacerations/abrasions, blisters, muscle strains, cramping and ankle sprains most commonly diagnosed. TR illness involved the gastrointestinal tract (GIT), metabolic and cardiovascular body systems. Symptoms of nausea and vomiting related to GIT distress and dehydration are commonly reported.

Conclusions Injury and illness are common among TRs participating in TR races. Limited evidence is available on trainingrelated injury and illness in TR specific.

\section{RELATIONSHIP OF PATELLOFEMORAL ANGLES AND TIBIOFEMORAL ROTATIONAL ANGLES WITH JUMPER'S KNEE IN PROFESSIONAL FOLK DANCERS: AN MRI ANALYSIS}

${ }^{1}$ Neslihan Aksu, ${ }^{1}$ Vefa Atansay, ${ }^{2}$ ssik Karalok, ${ }^{1}$ Ayhan Nedim Kara, ${ }^{3}$ Azmi Hamzaoglu. ${ }^{1}$ Demiroglu Bilim University Medical Faculty Florence Nightingale Hospital Orthopedics and Traumatology Department, Istanbul, Turkey; ${ }^{2}$ Demiroglu Bilim University Medical Faculty Florence Nightingale Hospital Radiology Department, Istanbul, Turkey; ${ }^{3}$ Istanbul Florence Nightingale Hospital Orthopedic and Spine Center, Istanbul, Turkey

\subsection{6/bjsports-2021-IOC.305}

Background Professional dancers learn splash and landing techniques throughout their careers starting in childhood and practice it very frequently like basketball, volleyball and soccer. Among the intrinsic factors, anatomical features of the lower extremity were the most studied in the literature.

Objective In this article, we investigated the relationship of tibiofemoral rotational angles and patellofemoral (PF) angles to the development of jumper's knee in professional folk dancers.

Design Retrospective cohort MRI study.

Setting Professional folk dance group.

Patients (or Participants) 26 professional folk dancers (16 male, 10 female; mean age of $30.69 \pm 7.51$ years (17 to 46$)$ ) group with complaints of knee pain.

Interventions (or Assessment of Risk Factors) PF sulcus angle and Femur-Insall angle were found to be related to Jumper's knee.

Main Outcome Measurements We examined 26 dancers with complaints of knee pain, and 32 knees of them had magnetic resonance imaging (MRI). We detected 21 jumper's knees. We measured patellofemoral angles (Patellofemoral sulcus angle, Lateral patellofemoral angle, Patellar tilt angle, Lateral trochlear-inclination angle, Lateral patellar tilt angle, The patellofemoral congruence angle) and tibiofemoral rotational angles (Condillary twist angles, posterior condillary angles, femurInsall angles, tibia-Insall angles, posterior tibiofemoral angles, Whiteside-PFCL angles) and noted patellar specifics as alta, Baja, Wiberg on MRI's with and without jumper's knee to understand if there is any relationship with tendinopathy occurrence in this cohort study.

Results According to logistic regression analysis, PF sulcus angle was found to be related to quadriceps tendinopathy development $(\mathrm{p}<0,05$, odd ratio (OR): 1,24, 95\% confidential interval (CI ): 1,03-1,5) and patellar tendinopathy is found to be related to Femur-Insall angle $(\mathrm{p}<0,05$, OR: 1,27, 95\% CI: $1,00-1,61)$.
Conclusions The patellofemoral sulcus angle and patellar tendon rotation relative to the femur may be the effective anatomical variations in jumper's knee occurence.

\section{CALCANEAL BONE DENSITY AND BONE STRESS INJURY IN NCAA DIVISION I ATHLETES AND NON- INTERCOLLEGIATE ATHLETE COLLEGE STUDENTS}

${ }^{1}$ Jason Bennett, ${ }^{2}$ Tricia Austin, ${ }^{2}$ Ann Hayes, ${ }^{3}$ Mark Reinking. ${ }^{1}$ Carroll University, Waukesha, USA; ${ }^{2}$ Saint Louis University, Saint Louis, USA; ${ }^{3}$ Regis University, Denver, USA

\subsection{6/bjsports-2021-IOC.306}

Background There is limited evidence describing the relationship between calcaneal bone mineral density (cBMD) and activity level or lower extremity overuse bone injury (LEOBI). Objective The purposes of this study were to: 1) compare cBMD of intercollegiate athletes (ICA) and non-intercollegiate athlete (NA) college students, 2) examine the influence of physical activity on cBMD in NA, and 3) determine if there is an association between $\mathrm{CBMD}$ and the development of LEOBI.

Design Prospective, cohort study.

Setting NCAA Division I University.

Participants 84 ICA and 103 NA college students.

Assessment ICAs provided injury and menstrual history, were measured for $\mathrm{CBMD}$ at the beginning and end of the year, and were followed for occurrence of LEOBI. NA college students provided injury and menstrual history and were measured for cBMD.

Main Outcome Measures Descriptive statistics, statistical analyses of relationships, logistic regression, and t-tests were used in the statistical analyses.

Results Eight ICAs were diagnosed with a LEOBI over the year. There was no difference in initial cBMD between ICAs with and without LEOBI; right $(\mathrm{p}=.05)$ and left $\mathrm{cBMD}$ $(p=.07)$ were lower in those ICAs with LEOBI at the end of the season. The NAs had significantly lower cBMD and speed of sound (SOS) than the ICAs. There were no significant differences in $\mathrm{CBMD}$ and SOS values between the 8 ICAs with LEOBI and the 103 NAs. For the NAs, there was no significant correlation between cBMD and activity, however, age of onset of menstruation and $\mathrm{CBMD}$ were found to be significantly correlated $(\mathrm{p}<.05)$.

Conclusions cBMD was significantly lower in NAs as compared to ICAs. The ICAs with LEOBI did not have significantly different cBMD than the NAs. The difference in cBMD between ICAs and NAs may be activity related, but differences in cBMD among the NAs was not related to activity level.

\section{HIP EXTENSOR WEAKNESS IS ASSOCIATED WITH LOWER LIMB MUSCLE STRAIN IN MALE ELITE VOLLEYBALL ATHLETES}

${ }^{1}$ Natália FN Bittencourt, ${ }^{1}$ Renato de Paula da Silva, ${ }^{1}$ Paola de Figueiredo Caldeira, ${ }^{2}$ Alysson Lima Zuin, ${ }^{2}$ Daniel Bornelli Campos Serio, ${ }^{2}$ Petterson Moura da Silva, ${ }^{1,3}$ Luciana De Michelis Mendonça. 'PHAST - Physiotherapy Assessment Tool, Belo Horizonte, Brazil; ${ }^{2}$ Sada Cruzeiro Vôlei, Belo Horizonte, Brazil; 3UFVIM - Universidade Federal dos Vales do Jequitinhonha e Mucuri, Diamantina, Brazil

10.1136/bjsports-2021-IOC.307 